\title{
Unilateral rhinophyma: report of a case and review
}

\author{
Scott Walter ${ }^{1}$, Steven Krueger ${ }^{2}$, Jonathan Ho', Kavitha K. Reddy ${ }^{1}$ \\ ${ }^{1}$ Department of Dermatology, Boston University School of Medicine, Boston, MA 02118, USA. \\ ${ }^{2}$ University of Massachusetts Medical School, 55 N Lake Ave, Worcester, MA 01655, USA.
}

Correspondence to: Dr. Kavitha K. Reddy, Department of Dermatology, Boston University School of Medicine, Boston, MA 02118, USA.

E-mail: kreddy@bu.edu

How to cite this article: Walter S, Krueger S, Ho J, Reddy KK. Unilateral rhinophyma: report of a case and review. Plast Aesthet Res 2017;4:49-53.

\section{Article history: \\ Received: 08-02-2017 \\ Accepted: 14-03-2017 \\ Published: 22-03-2017}

Key words:

Rosacea,

rhinophyma,

unilateral,

phymatous rosacea

\section{ABSTRACT}

Rhinophyma is a less-common subtype of rosacea that presents as thickened skin with enlarged sebaceous glands that may progress to large bulbous growths with dilated pores on the nose. Rhinophyma can lead to morbidity aesthetically and sometimes functionally. The prevalence of rosacea ranges from $1 \%$ to $20 \%$. The exact pathogenesis is not known, but potential factors include altered circulation, changes in microorganisms and/or alterations in immunity. Here the authors present a unique case where a patient presented with unilateral rhinophyma: a presentation warranting work up to rule out other more worrisome entities.

\section{INTRODUCTION}

Rhinophyma is the most prevalent presentation of the phymatous subtype of rosacea. Rosacea subtypes include erythematotelangiectatic type (type 1), papulopustular rosacea (type 2), phymatous rosacea (type 3) and ocular rosacea (type 4). ${ }^{[1]}$ Phymatous rosacea is characterized early by prominent follicular pores or patulous follicles with mild swelling while advanced disease reveals pronounced hyperemic skin thickening, irregular surface nodularities representing sebaceous gland hypertrophy and eventual distortion of the nasal surface architecture. ${ }^{[2]}$ It typically occurs on the nose as a bulbous irregular growth with dilated pores and background telangiectasia. Occasionally very advanced disease may lead to nasal obstruction and sleep apnea. ${ }^{[3]}$ While rhinophyma refers to when this subtype occurs on the nose, phymatous rosacea can also occur more rarely on the chin (gnathophyma), ears (otophyma), forehead (mentophyma), or eyelids (blepharophyma). Rhinophyma can occur in isolation and its severity does not always correlate with duration of disease. ${ }^{[4]}$ It is no longer thought to be an end stage of rosacea. ${ }^{[2]}$

Here we present a case of a patient with unilateral rhinophyma of the right nasal ala. This is an unusual presentation for which we believe this is the first report, as we cannot find any other reports in the literature.

\section{CASE REPORT}

A 77-year-old male with a medical history of hypertension and chronic kidney disease presented 
with a 1-year history of a growing mass on the right nasal ala. The patient reported it started as a small papule and spread over the ala over the course of a year; he noticed most of the growth during the last 4 months up to presentation. The patient denied any history of trauma to the area and denied manipulating the area. He denied using any topical medications or products on his nose. He did not have a history of similar lesions on the nose in the past. He denied any personal or family history of rosacea or of skin cancer. The patient was originally from El Salvador and then immigrated to the United States. He was retired from his work at the time of presentation. He previously worked outdoors in construction for many years and had a number of sunburns in the past.

Physical examination revealed a $2.5 \mathrm{~cm} \times 2.0 \mathrm{~cm}$ soft lobulated skin colored nodule with overlying prominent dilated pores encompassing the entire right nasal ala [Figure 1]. The left nasal ala was not affected. On examination of the remainder of his face, his bilateral cheeks and nose showed sebaceous skin with multiple scattered dilated pores and open comedones and a few small telangiectasias. There were no facial pustules. There was not any palpable lymphadenopathy. The remainder of his skin on his body was normal. Because the differential diagnosis could include cutaneous sarcoidosis, we asked the patient and he did not have a cough or any shortness of breath. Review of systems was negative for any other symptoms or concerns. Given the growth and unilateral nature of the identified nodule, a shave biopsy was performed on the edge of the mass to evaluate the lesion. The pathology report from the biopsy was read as a fibrous papule. Clinically, however, the lesion was more consistent with rhinophyma. The patient underwent electrosurgical excision of the growth. The site healed successfully with secondary intention and a restored normal nasal alar contour [Figure 2]. Final excision pathology was consistent with rhinophyma [Figure 3]. The patient agreed with taking doxycycline $20 \mathrm{mg}$ orally twice daily indefinitely as an anti-inflammatory treatment for rosacea and to attempt to prevent recurrence. He has maintained his results 1 year later.

\section{DISCUSSION}

While the prevalence of rosacea overall is estimated to be from $1 \%$ to $20 \%$, the phymatous subtype is less common. ${ }^{[5]}$ In a population study of Estonian workers with Rosacea, only $1 \%$ was classified as having subtype 3. Rosacea overall has a slightly female predominance, but the incidence of rhinophyma is much higher in males and is seen most often after 40 years of age. ${ }^{[3,5,6]}$ Rosacea has been reported to be more frequent in skin phototypes I and II, though it is increasingly being recognized as a condition seen in all skin types. ${ }^{[2]}$

Although commonly diagnosed clinically, the differential diagnosis for rhinophyma should be considered, especially when appearing unilaterally as in our patient. Basal cell and squamous cell carcinomas can occur on phymatous skin, and should be considered when unilateral changes, rapid growth, ulceration or drainage occur. ${ }^{[2,3]}$ Other neoplasms including adnexal tumors would also be included and can be considered. Granulomatous processes such as sarcoidosis and infectious diseases such as rhinoscleroma (Klebsiella) or leishmania should also be considered in the appropriate clinical setting.

Histopathologically, rhinophyma classically shows findings compatible with rosacea (telangiectasia in the superficial dermis, dilated infundibula with occasional cysts and a lymphohistiocytic perifollicular infiltrate) with the addition of striking sebaceous hyperplasia. ${ }^{[4-7]}$ A severe form has also been described which shows marked dermal thickening with few infundibular cysts and reduction or absence of pilosebaceous structures. ${ }^{[4]}$

The exact pathogenesis of rosacea and rhinophyma is not known but it is thought to be a combination of multiple factors leading to vascular changes and a trigger of the innate immune system. Numerous vascular growth factors and receptors have been shown to be increased in affected skin leading to an overall state of abnormal vascular reactivity. Specifically, vascular endothelial growth factor (VEGF), VEGF receptors, lymphatic endothelium marker D2-40 and CD 31 expressions are increased which provide stimulants for proliferation of vascular and lymphatic endothelial cells. ${ }^{[8,9]}$ This correlates with the grossly irregular and dilated vascular networks seen in affected skin histopathologically. Sun or ultraviolet exposure is also considered a contributing factor. In mice, it has been shown that UVB light induces dermal angiogenesis and also increases VEGF expression in keratinocytes. ${ }^{[3]}$

Additionally, the innate immune response is triggered leading to an abnormal host response. Although the exact triggers are unknown, many environmental and genetic factors have been hypothesized to play a role. The cytokine cathelicidin has recently been found to be highly expressed in affected patients and thought to play a key role in the pathogenesis of rosacea. Triggered in response to innate antigens, this effector peptide has many functions including promoting angiogenic activity, modifying the local inflammatory response, regulating leukocyte chemotaxis and 

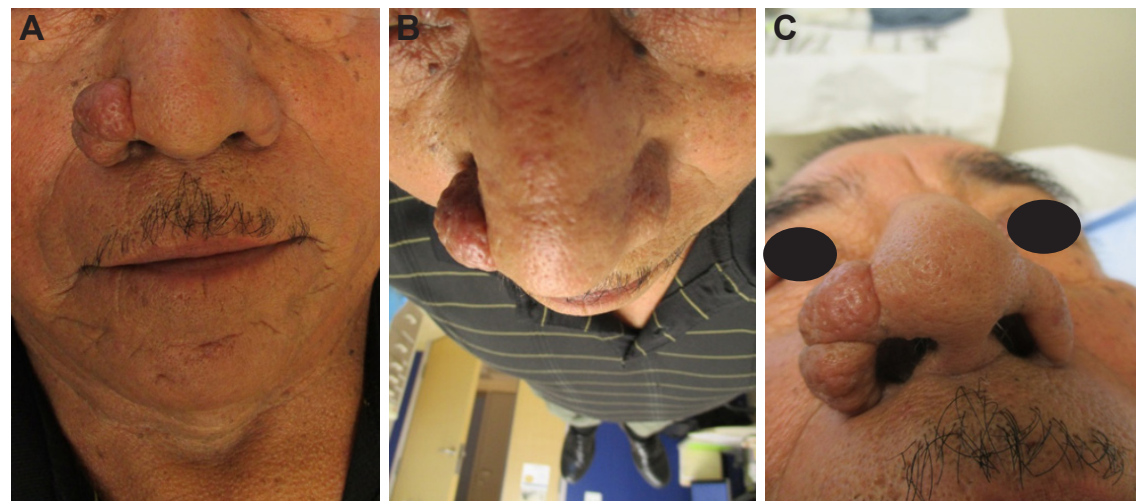

Figure 1: Unilateral rhinophyma (A: front view; B: superior view; C: inferior view)
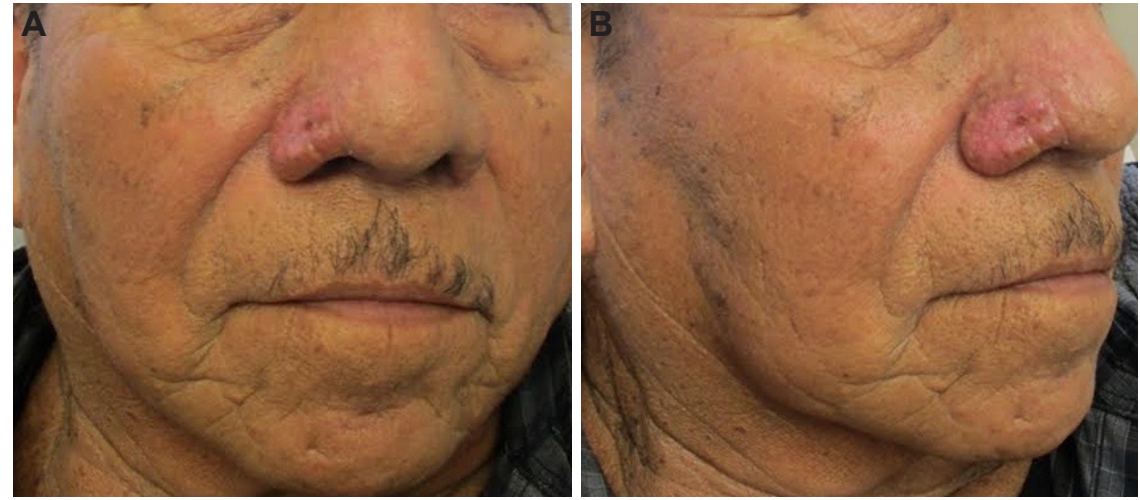

Figure 2: Unilateral rhinophyma 2 weeks after electrosurgical excision (A: front view; B: lateral view)

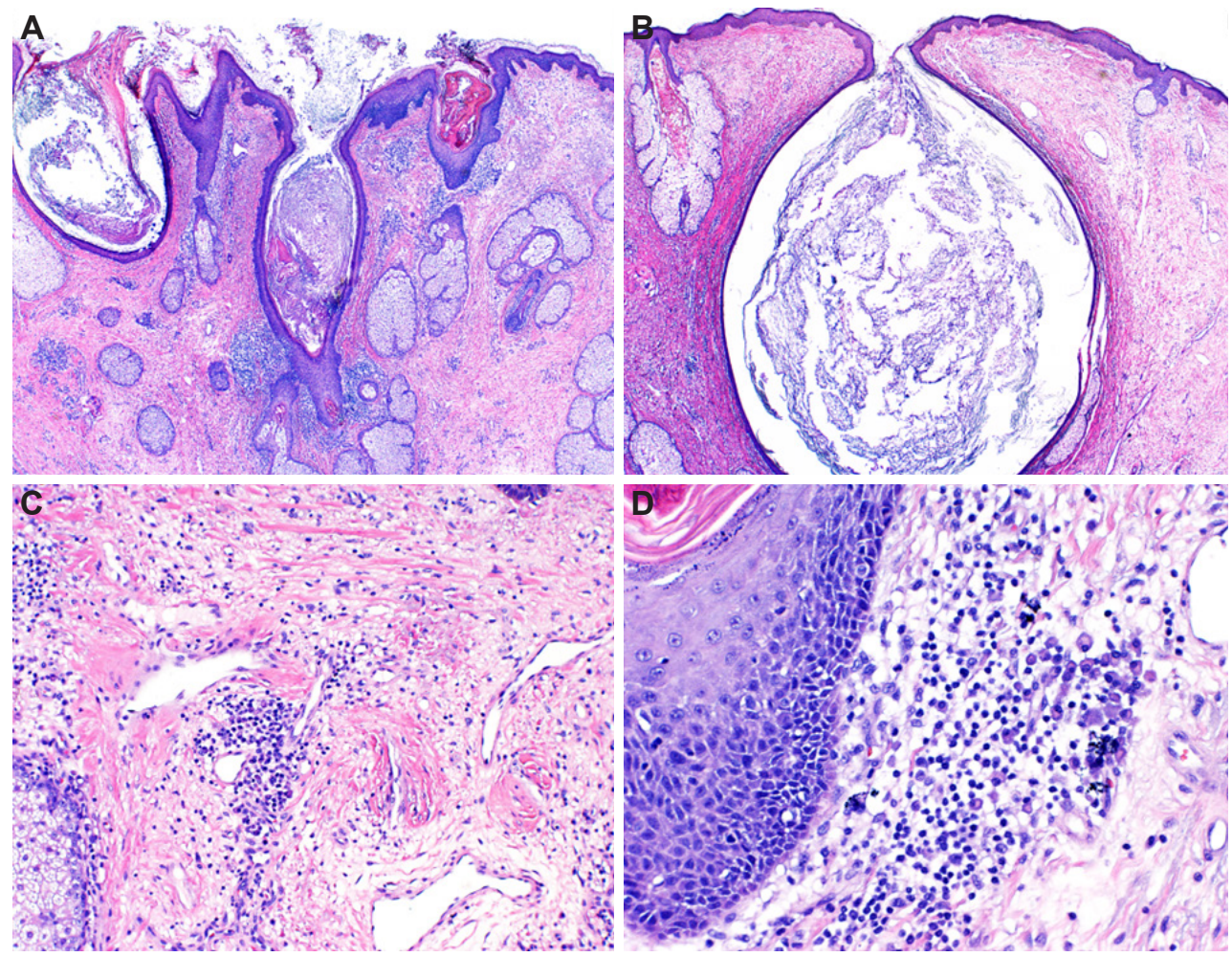

Figure 3: Histology of unilateral rhinophyma. Characteristic histopathologic features of advanced rhinophyma including comedonal (A) and cystic dilation (B) of follicular infundibulae, increased numbers of sebaceous glands (A), a perivascular lymphocytic infiltrate, marked dermal fibrosis and telangiectasia (C). A moderately dense perifollicular lymphocytic infiltrate with numerous plasma cells (D) typical of rosacea and its variants is also present. ( $\times 20$ in $A$ and $B ; \times 100$ in $C ; \times 200$ in D) 
increasing vascular permeability. ${ }^{[10,11]}$

What exactly triggers the innate immune response is not known but UV light, trauma and microorganisms such as Demodex mites are thought to play a role. Demodex mites, although common in the general population, are prevalent in up to $100 \%$ of rosacea patients. ${ }^{[1]}$ The mites are thought to trigger an immune response, act as a vector for other bacterial pathogens and block hair follicles. ${ }^{[3]}$ Additionally, smokers have been found to have a higher risk of developing rosacea. ${ }^{[12]}$

The exact pathogenesis of the phymatous stage of rosacea is also not well understood. It is postulated to be a combination of the above factors in addition to alterations in blood flow via decreased dermal vasoactive intestinal peptide receptors. ${ }^{[13]}$ In the severe variant of rhinophyma described by Aloi et al., ${ }^{[4]}$ they hypothesize that the pathogenesis is similar to that of lymphedema. They suggest that the severe fibroplasia seen in this form of rhinophyma impairs lymphatic drainage which leads to persistent edema and destruction of adnexal structures. Over time, this fluid collection stimulates further fibrosis via production of collagen and glycosaminoglycans.

The treatment for rhinophyma is approached in a different fashion compared to the other rosacea subtypes. Younger patients with early signs of rhinophyma may respond well to oral isotretinoin, but surgery remains the only definitive treatment for those with advanced disease..$^{[1,6,14,15]}$ A surgical approach may include electrocautery, laser ablation, cryotherapy, dermabrasion, paring with a scalpel blade, or a combination of these techniques to achieve an optimal outcome. ${ }^{[14,15]}$ Use of electrosurgery to excise the rhinophyma can reduce bleeding. ${ }^{[6]}$ Preservation of the sebaceous glands allows for adequate reepithelialization, and the excision should not extend deeply enough to expose cartilage. ${ }^{[6,15]}$ Skin grafts or local flaps can be used to cover the defect, or the wound can be allowed to re-epithelialize spontaneously within 2 weeks. Removing too much tissue can result in a smooth, shiny scar that does not match the rest of the nose. ${ }^{[6,15]}$ Laser ablation therapy is another option for treatment. One study evaluated 24 patients with rhinophyma who were treated with a 10,600-nm $\mathrm{CO}_{2}$ pulsed laser. They found that $79.1 \%$ had high improvement, $16.7 \%$ had moderate improvement and $4.2 \%$ had low improvement, with minimal side effects. ${ }^{[16]}$

Here we have presented what is, to our knowledge, the first reported case of unilateral rhinophyma. ${ }^{[17]}$ Why it appeared unilaterally is unclear. Interestingly, unilateral otophyma has been reported, and the authors postulated that it may have occurred unilaterally due to localized factors such as sleeping on the affected ear, trauma or infection. ${ }^{[17]}$ The etiology of unilateral rhinophyma remains unknown, and possibilities include spontaneous or idiopathic asymmetric inflammation, or a localized lymphedematous or inflammatory process, such as might result from localized infection or trauma. Our patient has healed well after electrosurgical treatment and is pleased with his improvement.

\section{Authors' contributions}

Concept design: K.K. Reddy

Definition of intellectual content: K.K. Reddy, S. Walter Literature search, data acquisition, data analysis: S. Walter, J. Ho

Manuscript preparation, editing, and review: S. Walter, J. Ho, S. Krueger, K.K. Reddy

\section{Financial support and sponsorship}

None.

\section{Conflicts of interest}

There are no conflicts of interest.

\section{Patient consent}

The proper consent of the patient was taken for carrying out all the tests and treatment.

\section{Ethics approval}

The procedures followed were in accordance with ethical standards of the responsible committee on human experimentation. As an experiment was not performed, local institutional review board protocol was not required.

\section{REFERENCES}

1. Crawford GH, Pelle MT, James WD. Rosacea: I. Etiology, pathogenesis, and subtype classification. J Am Acad Dermatol 2004;51:327-41

2. Powell FC, Raghallaigh SN. Rosacea and related disorders. In: Bolognia JL, Schaffer JV, Jorizzo JL. Dermatology. 3rd. Philadelphia: Elsevier Saunders; 2012. p. 561-70.

3. Tüzün Y, Wolf R, Kutlubay Z, Karakuş O, Engin B. Rosacea and rhinophyma. Clin Dermatol 2014;32:35-46.

4. Aloi F, Tomasini C, Soro E, Pippione M. The clinicopathologic spectrum of rhinophyma. J Am Acad Dermatol 2000;42:468-72.

5. Tan J, Berg M. Rosacea: current state of epidemiology. J Am Acad Dermatol 2013;69:S27-35.

6. Roberts JO, Ward CM. Rhinophyma. J R Soc Med 1985;78:678-81.

7. Marks R, Harcourt-Webster JN. Histopathology of rosacea. Arch Dermatol 1969;100:683-91.

8. Gomaa AH, Yaar M, Eyada MM, Bhawan J. Lymphangiogenesis and angiogenesis in non-phymatous rosacea. J Cutan Pathol 2007;34:74853.

9. Smith JR, Lanier VB, Braziel RM, Falkenhagen KM, White C, 
Rosenbaum JT. Expression of vascular endothelial growth factor and its receptors in rosacea. Br J Ophthalmoly 2007;91:226-9.

10. Reinholz M, Ruzicka T, Schauber J. Cathelicidin LL-37: an antimicrobial peptide with a role in inflammatory skin disease. Ann Dermatol 2012;24:126-35.

11. Lazaridou E, Apalla Z, Sotiraki S, Ziakas NG, Fotiadou C, Ioannides D. Clinical and laboratory study of rosacea in northern Greece. J Eur Acad Dermatol Venereol 2010;24:410-4.

12. Breton AL, Truchetet F, Véran Y, Doumat-Batch F, Baumann C, Barbaud A, Schmutz JL, Bursztejn AC. Prevalence analysis of smoking in rosacea. J Eur Acad Dermatol Venereol 2011;25:1112-3.

13. Sibenge S, Gawkrodger DJ. Rosacea: a study of clinical patterns blood flow, and the role of demodex folliculorum. $J$ Am Acad Dermatol 1992;26:590-3.

14. Pelle MT, Crawford GH, James WD. Rosacea: II. therapy. J Am Acad Dermatol 2004;51:499-512.

15. Lloyd KM. Surgical correction of rhinophyma. Arch Dermatol 1990;126:721-3

16. Bassi A, Campolmi P, Dindelli M, Bruscino N, Conti R, Cannarozzo G, Pimpinelli N. Laser surgery in rhinophyma. G Ital Dermatol Venereol 2016;151:9-16.

17. Alcántara-Reifs CM, Salido-Vallejo R, Garnacho-Saucedo G, Vélez García-Nieto A. Otophyma: a rare variant of phymatous rosacea. Am J Otolaryngol 2016;37:251-4. 